\title{
Chagas Disease: Still Many Unsolved Issues
}

\author{
José M. Álvarez, ${ }^{1}$ Raissa Fonseca, ${ }^{1}$ Henrique Borges da Silva, ${ }^{1}$ \\ Cláudio R. F. Marinho, ${ }^{2}$ Karina R. Bortoluci, ${ }^{3}$ Luiz R. Sardinha, ${ }^{4}$ \\ Sabrina Epiphanio, ${ }^{5}$ and Maria Regina D'Império Lima ${ }^{1}$ \\ ${ }^{1}$ Department of Immunology, Biomedical Sciences Institute, University of São Paulo, 05508-000 São Paulo, SP, Brazil \\ ${ }^{2}$ Department of Parasitology, Biomedical Sciences Institute, University of São Paulo, 05508-000 São Paulo, SP, Brazil \\ ${ }^{3}$ Department of Biological Sciences, UNIFESP (Campus Diadema), 09972-270 Diadema,SP, Brazil \\ ${ }^{4}$ Hospital Israelita Albert Einstein, 05652-000 São Paulo, SP, Brazil \\ ${ }^{5}$ Department of Clinical and Toxicological Analyses, Faculty of Pharmaceutical Sciences, University of São Paulo, \\ 05508-000 São Paulo, SP, Brazil
}

Correspondence should be addressed to José M. Álvarez; jmamosig@icb.usp.br

Received 22 April 2014; Accepted 15 June 2014; Published 29 June 2014

Academic Editor: Edecio Cunha-Neto

Copyright (C) 2014 José M. Álvarez et al. This is an open access article distributed under the Creative Commons Attribution License, which permits unrestricted use, distribution, and reproduction in any medium, provided the original work is properly cited.

\begin{abstract}
Over the past 20 years, the immune effector mechanisms involved in the control of Trypanosoma cruzi, as well as the receptors participating in parasite recognition by cells of the innate immune system, have been largely described. However, the main questions on the physiopathology of Chagas disease remain unanswered: "Why does the host immune system fail to provide sterile immunity?" and "Why do only a proportion of infected individuals develop chronic pathology?" In this review, we describe the mechanisms proposed to explain the inability of the immune system to eradicate the parasite and the elements that allow the development of chronic heart disease. Moreover, we discuss the possibility that the inability of infected cardiomyocytes to sense intracellular T. cruzi contributes to parasite persistence in the heart and the development of chronic pathology.
\end{abstract}

\section{A Brief Overview of Chagas Disease}

Chagas disease is caused by Trypanosoma cruzi and represents an important health problem in Latin America, with approximately 8 million chronically infected people [1]. Recently, as a consequence of human migrations, Chagas disease has become a potential public health issue in developed countries, and significant increases in confirmed cases have been reported in the USA, Canada, Europe, Japan, and Australia [2]. The invasion of the human host frequently occurs by the passage through damaged skin or intact mucosa of metacyclic trypomastigotes released with the feces of infected triatomines after their blood meal. Alternatively, infection through other routes, such as oral, congenital, and blood transfusion/organ transplantation, also occurs. Because it is an obligate intracellular parasite, $T$. cruzi can be found in the vertebrate host as amastigotes, the intracellular replicative form, and as extracellular trypomastigotes circulating freely in the blood and tissues. The infection has a self-limiting acute phase, with patent (or subpatent) parasitemia, which goes unnoticed in many infected individuals. At this stage, the parasites actively replicate in many different cell types, such as macrophages; smooth, striated, and cardiac muscle cells; adipocytes; and cells of the central nervous system [3].

While a small proportion of patients succumbs to the acute phase of the disease, the development of the adaptive immune response typically provides control of the T. cruzi infection, albeit nonsterile control. Failing to completely eradicate the parasite, individuals remain infected for life and establish a dynamic equilibrium with the parasite that results in different clinical outcomes. Thus, while many chronically infected individuals remain in the asymptomatic indeterminate phase, a significant proportion (30-35\%) of patients develop the cardiac or digestive manifestations of chronic disease: cardiomyopathy that may lead to congestive heart failure, arrhythmia and, eventually, patient death, and esophageal or colonic megasyndromes. These are irreversible pathological changes that occur despite parasite scarcity. 
Recapitulating human chagasic myocarditis, mice surviving long-term infection by certain stocks of T. cruzi develop chronic myocardial lesions $[4,5]$.

\section{The Host Immune Response against $T$. cruzi}

The immune system is well equipped to detect and control T. cruzi parasites through the combined effect of diverse branches of the immune response. $\mathrm{CD}^{+}$and $\mathrm{CD} 8^{+} \mathrm{T}$ cells, as well as $\mathrm{B}$ cells, contribute to control the parasite through cytokine secretion, cellular cytotoxicity, and specific antibody production [6-8]. From the end of the acute phase and throughout chronic infection, T. cruzi-specific IgG antibodies actively participate in the removal of extracellular parasites released from ruptured tissue nests, an effect that presumably occurs by promoting parasite phagocytosis by macrophages and neutrophils. In addition, specific IgG antibodies mediate the removal of blood trypomastigotes, a clearance process in which complement and mononuclear phagocytes from the liver, spleen, and lungs appear to be involved [9-11]. The IFN- $\gamma$ produced by activated $\mathrm{CD}^{+}$and $\mathrm{CD}^{+} \mathrm{T}$ cells, NK cells, and $\mathrm{CD} 4^{-} \mathrm{CD} 8^{-} \gamma \delta$ T cells plays a crucial role in parasite elimination $[12,13]$. IFN- $\gamma$ potentiates the effector activity of macrophages by inducing the transcription of the inducible nitric oxide synthase (iNOS) gene, notably increasing the production of nitric oxide, which has a potent effect on T. cruzi killing $[14,15]$. In addition, IFN- $\gamma$ promotes the immunoglobulin switch to IgG subclasses with high opsonizing and complement-activating potential. Lastly, cytotoxic $\mathrm{CD}^{+}{ }^{+} \mathrm{T}$ cells also contribute to T. cruzi control through the recognition and destruction of cells that harbor intracellular forms of the parasite [16].

\section{Limitations to the Innate and Acquired Immune Responses That Contribute to Parasite Persistence}

One of the most intriguing questions of human and experimental T. cruzi infection is why the immune system fails to totally eradicate the parasite. At first glance, the inability of the infected host to attain sterility suggests that the immune effector activity directed against the parasite is insufficient or inappropriate due to defective activation of the specific immune response or excessive regulation of this response.

In this context, we outline in this section the different escape mechanisms employed by T. cruzi parasites and discuss the hypothesis generated to explain an immune system failure. At the beginning of the infection (before development of the parasite-specific response), T. cruzi trypomastigotes escape lysis by the complement system, an evasion strategy that results from the presence of complement-regulatory molecules on the parasite surface [17]. In addition, internalized parasites of diverse T. cruzi strains escape the phagocytic vacuole of unprimed resident macrophages [18], a strategy that relies on a variety of molecules with antioxidant properties $[19,20]$. Nevertheless, as infection progresses, these two evasion strategies are largely circumvented by the development of the specific humoral response and the induction of macrophage activation by IFN- $\gamma$ and other cytokines. Because T. cruzi strains display different levels of antioxidant activity that directly correlate with strain virulence [21], it remains unclear whether IFN- $\gamma$ confers effective macrophage protection against any T. cruzi parasite or results in different degrees of intracellular parasite destruction for different isolates.

Pattern recognition receptors (PRRs), such as toll-like receptors (TLRs) 2, 4, 7, and 9, nucleotide-binding oligomerization domain-like receptor (NOD) 1 and NATCH, LRR, and PyD domains-containing protein 3 (NLRP3) have been shown to participate in T. cruzi detection by macrophages and dendritic cells [22-27]. However, a deficient innate immune response due to poor PRR signaling by pathogen-associated molecular patterns (PAMPs) has been proposed as a mechanism involved in parasite escape. This hypothesis is supported by data showing that, compared with mice infected by wildtype T. cruzi parasites, mice infected with transgenic T. cruzi expressing Salmonella typhimurium flagellin (fliC) display an increased innate response mediated by macrophages and dendritic cells and notably enhanced adaptive immunity [28]. More importantly, in the chronic phase, mice infected with fliC-transgenic T. cruzi display notably reduced parasite levels in relation to those infected with wild-type parasites.

Parasite persistence has also been attributed to the relatively slow development of $T$. cruzi-specific $\mathrm{CD}^{+} \mathrm{T}$ effector cells [29], a phenomenon explained by diverse factors, including the postulated poor PAMP activity of T. cruzi [28] and the induction of a strong Fas expression on T. cruzispecific $\mathrm{CD}^{+} \mathrm{T}$ cells [30]. Another possible reason for the failure to acquire sterile immunity is the clonal dominance of the lymphocyte response to T. cruzi infection. This is illustrated by the observation that the $\mathrm{CD}^{+} \mathrm{T}$ cell response to the abundantly expressed T. cruzi antigen amastigote surface protein-2 (ASP2) is restricted to a small number of clones [31]. Narrowing the scope of parasite peptides that are recognized by $\mathrm{CD}^{+} \mathrm{T}$ cells may impair complete parasite eradication during chronic infection and the control of reinfection, which frequently occurs in endemic areas.

An alternative mechanism that restricts the efficiency of adaptive immunity in eliminating $T$. cruzi parasites is the negative regulation of effector lymphocytes because of persistent stimulation. The senescence of $\mathrm{CD} 4^{+} \mathrm{T}$ cells [32] and exhaustion of $\mathrm{CD}^{+} \mathrm{T}$ cells that infiltrate the heart or striated muscle of chronically infected hosts have been observed during chronic infection $[33,34]$. In this context, it was suggested that the parasite can survive inside myocardial or striated muscle cells because following migration to the tissues $\mathrm{CD}^{+} \mathrm{T}$ cells lose their cytotoxic and IFN- $\gamma$-producing capacities [35]. Also suggesting the negative regulation of the effector response, Albareda and cols [36] reported that $\mathrm{CD} 4^{+}$ $\mathrm{T}$ cells from patients with long-term chronic infection are primarily monofunctional, whereas in children in the early chronic stage of infection, multifunctional responses are also observed. Last, the expression of PD-1 and PD-L1 regulatory molecules has been shown to downmodulate the effector activity of $\mathrm{CD}^{+}$and $\mathrm{CD}^{+} \mathrm{T}$ cells in T. cruzi-infected mice [37]. While PD-L1-PD-1 regulatory interaction was observed in the acute phase of infection, its roles in limiting parasite 

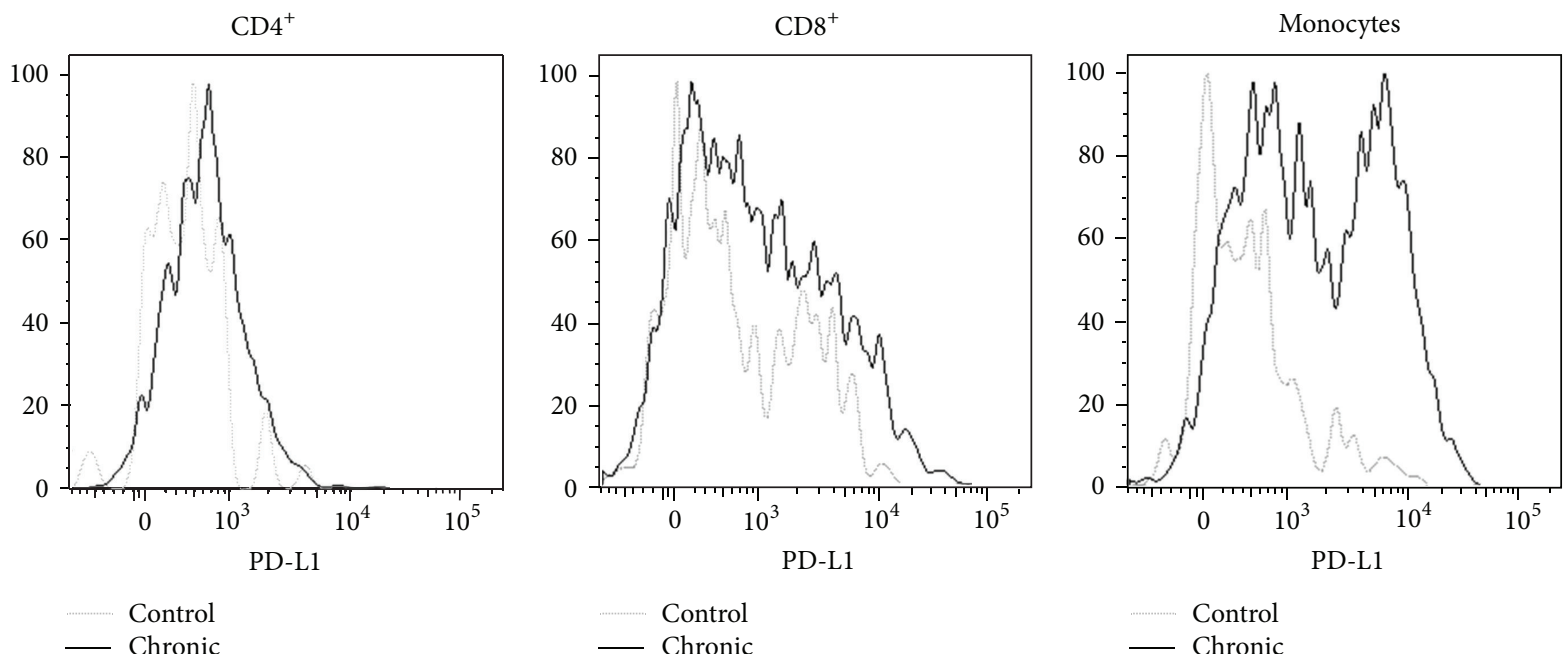

FIGURE 1: Expression of PD-L1 by infiltrating leukocytes in a chronically infected heart. C3H/HePAS mice were infected with $4 \times 10^{5}$ Sylvio X10/4 trypomastigotes obtained from LLCMK2 cultures. At day 400 postinfection, mice were sacrificed, and the heart tissue was digested with collagenase to isolate the infiltrating leukocytes. PD-L1 expression was analyzed by flow cytometry. Heart leukocytes from a pool of age-matched noninfected mice were also included.

elimination and permitting the perpetuation of lesions in the chronic phase remain to be determined. In this respect, our data showing the intense expression of PD-L1 in the heart-infiltrating leukocytes of mice chronically infected by Sylvio X10/4 T. cruzi parasites suggest the involvement of this regulatory circuit in parasite persistence (Figure 1).

The mechanisms of parasite escape discussed above refer to limitations of the innate or acquired protective immune response to T. cruzi that appear to operate in all infected individuals. However, in addition to these general elements, we must consider that individuals differ in the intensity/effectiveness of their anti-T. cruzi humoral and cellular responses, a consequence of polymorphisms in genes associated with the immune response [38]; these polymorphisms influence the intensity of the anti-T. cruzi effector activity, yielding different levels of residual parasites and undesired tissue lesions in chronically infected human patients. In addition, these polymorphisms influence the residual parasite distribution in different tissues/organs, an element closely connected to the development of the different forms of the disease.

The isogenic strains of mice also differ in the immune response to $T$. cruzi. These differences are often critical for acute phase survival [39] and most likely determine the parasite load in the chronic phase. However, not a single mouse strain has been reported to promote the complete elimination of the parasite. Therefore, regardless of the mouse/parasite strain combination, the inevitable outcome of murine infection by $T$. cruzi appears to be parasite persistence, a result analogous to that observed in human patients.

\section{Only a Proportion of T. cruzi-Infected Individuals Show Chronic Pathologies}

A significant proportion of chronically infected individuals develop the cardiac and digestive forms of the disease, but the largest fraction present the indeterminate form. Importantly, although many indeterminate patients remain asymptomatic for the rest of their lives, it is estimated that, each year, $2.5 \%$ of infected individuals evolve from the indeterminate to the clinical forms [40]. Chronic chagasic cardiomyopathy (CCC) represents the main cause of death in T. cruzi-infected patients. Moreover, this clinical form represents an important social burden in terms of lost labor hours and hospital costs. The situation of CCC patients is worrisome because the specific anti-T. cruzi drugs, currently limited to benznidazol and nifurtimox, show limited efficacy in chronically infected patients.

Because of parasite scarcity in the inflamed heart, CCC was long considered an autoimmune disease directed against self-epitopes showing cross-reactivity with parasite antigens [41]. According to this view, lesions were thought to occur as a result of $\mathrm{T}$ cell reactivity against myosin and other heartderived proteins [42] as well as humoral reactivity to the beta-1-adrenergic and M2 cholinergic receptors, leading to autonomic nervous system imbalance [43]. However, in the last 20 years, cumulative evidence has promoted a change in our understanding of this process. First, immunohistochemistry data showed that, in patients with CCC, the level of T. cruzi antigen correlated with the intensity of the inflammatory infiltrate [44]. Furthermore, T. cruzi DNA was found in the heart of diseased CCC patients but not in the heart of patients with the indeterminate form [45]. In contrast, patients with megaesophagus, one of the digestive forms of the disease, displayed positive PCR for T. cruzi kinetoplast DNA in the esophagus [46]. Confirming the human studies, in mice chronically infected by T. cruzi, we previously observed that live parasites were only detected in the heart of mice with cardiomyopathy, although these mice displayed subpatent blood parasite levels similar to those in mice with no heart pathology [47]. Based on the data from these and other important reports, at present, it is largely 


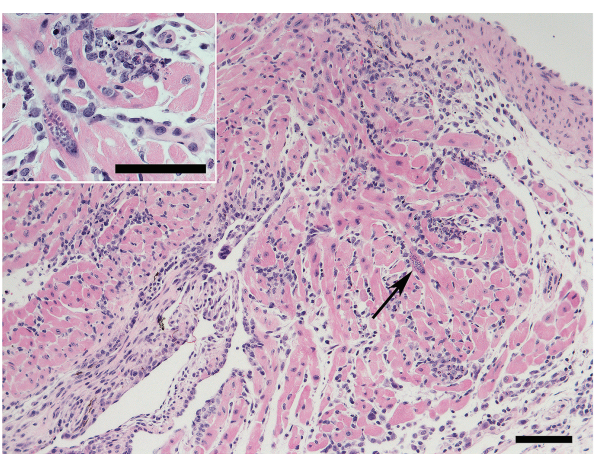

(a)

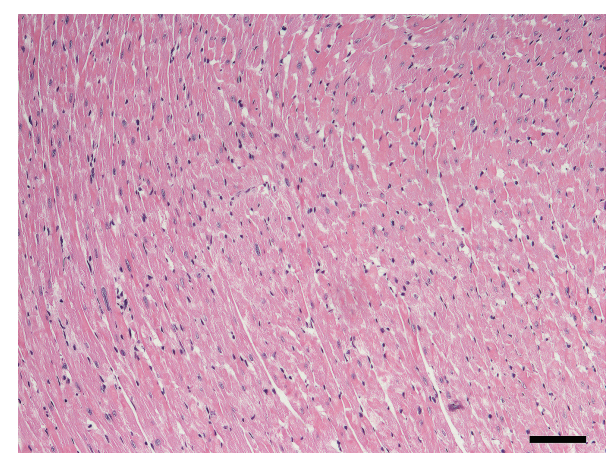

(b)

Figure 2: Parasite persistence is not the necessary outcome of heart infection by T. cruzi parasites. C57BL/6 mice were infected with $10^{3}$ T. cruzi blood trypomastigotes of the Y strain. At days 18 (a) and 180 (b) postinfection, mice were sacrificed, and the heart tissue was formalinfixed, included in paraffin, and stained with hematoxylin/eosin. Arrow in the figure shows an amastigote nest, which is magnified at the insert. Bars in figures correspond to $200 \mu \mathrm{m}$ and to $100 \mu \mathrm{m}$ at the insert.

agreed that the inflammatory infiltrate in CCC is caused by a response directed toward locally persisting parasites [48]. Still, because Chagas disease is a highly heterogeneous process influenced by host genetics, we cannot discard that the immune response in the heart of CCC patients could include, besides leukocyte reactivity towards locally persisting parasites, different degrees of autoreactivity. Furthermore, it is possible that a proportion of CCC patients shows cardiac pathology in the absence of locally persisting infection. This last possibility is illustrated by a recent report using bioluminescence imaging, in which mice chronically infected with luciferase expressing T. cruzi parasites display mild heart inflammation in the absence of local parasitism [49]. Further studies on the contribution of autoimmunity are required for a full comprehension of CCC physiopathology. These studies may eventually reveal alternative therapeutic approaches to attenuate heart tissue inflammation in chagasic patients.

Nonetheless, because most data suggest the association of chronic cardiac pathology with local parasite persistence, CCC is presently understood as an expression of the host's incapacity to totally eliminate the heart parasitism, a particularity of the host's failure to eradicate T. cruzi from the organism. This leads us to question why heart parasitism occurs in only a fraction of $T$. cruzi chronically infected individuals.

\section{Where Does T. cruzi Persist in the Chronically Infected Host?}

The tissue distribution of T. cruzi parasites varies among chronically infected individuals. As indicated above, patients with cardiomyopathy and megaesophagus harbor parasites in the heart and esophagus, respectively. However, are there in these patients, as well as in those with the indeterminate form, other locations where the parasite is relatively safe from complete elimination by the immune system? A priori, because parasite persistence is the rule, it is reasonable to hypothesize that immune-privileged T. cruzi reservoirs must exist in all chronically infected hosts. One of these parasite niches could be the central adrenal vein, which, in autopsy studies of patients with chronic Chagas disease, has been found to frequently harbor amastigote nests; further, the presence of these nests shows a close correlation with heart pathology parameters such as the intensity of leukocyte infiltration and myocardial fibrosis [50]. Additionally, both brown and white adipose tissues, as well as the colon and stomach, have been described as locations where T. cruzi parasites chronically persist [49, 51, 52]. Moreover, because of parasite persistence in the cardiac tissue of CCC patients, the heart could be one of these niches for a fraction of the infected population.

Another issue is whether parasite dissemination through the blood or by spreading from neighboring tissues occurs in the chronic host. Are the T. cruzi found in the hearts of CCC patients the consequence of chronic phase dispersion from other tissues or do they result from the perpetuation of heart colonization during the acute phase? This is an important question because chronic phase reinvasion could readdress the problem of heart parasite persistence outside the cardiac tissue.

On the other hand, it is important to note the observation made by different research groups that mice from certain strains can eliminate T. cruzi from the heart. This occurs in C57BL/6 mice infected with a sublethal dose of Y strain parasites; these mice exhibit strong leukocyte infiltration with the presence of amastigote nests in the heart in the late acute phase but no signs of infection or pathology in this organ in the chronic phase (Figure 2). This observation indicates that parasite persistence is not the necessary outcome of heart infection. Moreover, these results open the possibility that the indeterminate group of human patients might include patients in whom the cardiac infection was resolved in addition to individuals in whom the heart was never colonized by the parasite.

\section{Elements Involved in Parasite Persistence in the Heart or Other Tissues: Parasite Tropism}

T. cruzi displays a broad heterogeneity, being currently classified in six different groups (I-VI) [53] that show discrete 
correlations with the sylvatic or peridomestic forms of transmission as well as with the occurrence (or lack thereof) of different chronic pathologies. Mixed infections by multiple T. cruzi isolates are frequently observed in chronic chagasic patients.

Parasite tropism was originally defined as the preferential invasion of a cell type by a T. cruzi clone/isolate. Nevertheless, because the infected host exhibits considerable variability in its tissue responses to the parasite, tropism has to be redefined as the outcome of the interaction of a defined T. cruzi clone/isolate in a particular individual, a process largely dependent on the parasite and host genetics. The importance of the host in the development of chronic heart pathology by a single T. cruzi parasite is clearly illustrated in the murine model of infection by parasites of the T. cruzi clone Sylvio X10/4, which results in chronic cardiomyopathy in $\mathrm{C} 3 \mathrm{H} / \mathrm{HePAS}$ mice but not in $\mathrm{C} 57 \mathrm{BL} / 6$ or $\mathrm{A} / \mathrm{J}$ mice [47].

Therefore, while (at least for mice and men) the lack of a spontaneous cure and, consequently, the persistence of the parasite are general problems associated with $T$. cruzi infection, independent of parasite and host diversity, the development of chronic heart disease appears to be limited to particular host-parasite combinations.

\section{Is Parasite Persistence Merely the Result of a Deficit in the Local Immune Response?}

Different research groups have analyzed the heart-infiltrating leukocytes of CCC patients and mice with chronic cardiomyopathy [54-58]. These studies have yielded valuable data regarding the distribution of leukocyte populations, surface marker expression, and the production of cytokines, chemokines, and other mediators. Nonetheless, the gathered information did not help to determine whether a local immune deficit exists because the characteristics of an effective local immune response are undefined. This is because in those chronic settings in which an effective response could eventually occur, such as in chronically infected mice with no cardiac pathology or in patients with the indeterminate form, there are by definition no heart infiltrates to dissect. Therefore, if there is a defect in the local immune response in the heart of CCC patients, it has yet to be found.

Immune response analysis of the blood of patients with the cardiac and indeterminate forms has been used as an indirect means of searching for the presumed local immune defect, aiming to reveal a special immune signature that would explain why the parasite persists in the heart of CCC patients. Remarkably, these studies have revealed that the production of IFN- $\gamma$ and other proinflammatory cytokines is higher in CCC patients than in indeterminate form patients [59-61] and that asymptomatic patients exhibit augmented $\mathrm{T}_{\text {REG }}$ numbers and IL-10 levels $[59,62]$. Because a proinflammatory response is considered the appropriate approach to eliminate T. cruzi parasites, these results conflict with the hypothesis that parasite evasion is the result of a deficient immune response. Furthermore, while systemic studies do not suggest a local deficit in the anti-T. cruzi immune response of CCC patients, they do indicate that these patients

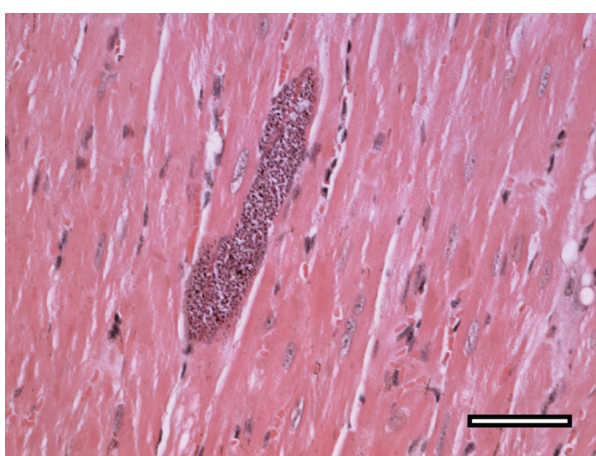

FIGURE 3: Lack of sensing of amastigote nests could contribute to parasite perpetuation and pathology in the chronically infected heart. Lack of sensing could occur independently of the origin of cardiomyocyte-invading trypomastigotes, that is, from a local ruptured nest or metastasis from a distant niche. The heart picture shown corresponds to a $\mathrm{C} 3 \mathrm{H} / \mathrm{HePAS}$ mouse infected for more than 200 days with $10^{6}$ Sylvio X10/4 trypomastigotes. The tissue section was stained with hematoxylin-eosin. Bar corresponds to $100 \mu \mathrm{m}$ (reproduced from C. R. F. Marinho, Microbes and Infection [64] with permission from Elsevier).

display an aggressive deregulated response that may explain the presence of tissue damage in the affected heart [63].

What, then, is missing? How can we explain why patients with greater proinflammatory responses to T. cruzi are those with lower heart parasite control? An interesting possibility is that T. cruzi persistence in the heart (and in other relatively immunoprivileged locations) is not merely the consequence of a defective local immune response but that it, to a large extent, derives from the parasite's ability to remain unnoticed inside the structural cells, safe from the effector activity of cytotoxic $\mathrm{CD}^{+} \mathrm{T}$ cells [64]. Illustrative evidence of this possibility is shown in Figure 3, where an amastigote nest can be observed amid myocardial fibers in a mouse infected for more than 200 days, that is, in an animal with a high level of anti-T. cruzi immune effector activity. While a notable feature of this picture is the nest size, the most impressive aspect is the absence of leukocytes surrounding it. This suggests a deficiency in signaling for leukocyte recruitment, a condition that allows the parasite to temporarily evade the immune system. Anecdotally, the presence of heart amastigote nests undetected by the inflammatory response was described by Vianna [65] in 1911, one year after the discovery of Chagas disease. From these observations, we can postulate that, independently of the cardiomyocyte-invading parasites originating from a local ruptured nest or a distant niche, the failure to attain sterile immunity in the heart may to a large extent result from an intrinsic or acquired deficit of the cardiomyocytes in sensing the intracellular parasite. Because leukocyte recruitment requires infected cardiomyocytes to signal the presence of an infection through the secretion of chemotactic molecules, we can speculate that, in patients with chronic cardiac disease, this mechanism is largely suppressed. Meanwhile, T. cruzi-infected neonatal cardiomyocytes have been shown in vitro to transcribe the genes for the chemokines MCP-1, RANTES, KC/GRO, MIP2, MIG, and IP-10 together with those for the cytokines 
TNF- $\alpha$ and IL-1 $\beta$ [66]. A way to reconcile these in vitro and in vivo findings is that the failure of cardiomyocyte signaling in vivo could be an adaptive trait that develops during the course of infection in only certain parasite-host combinations. The possibility that T. cruzi interferes with the physiology of infected structural cells is supported by other studies. In this manner, cruzipain, an enzyme abundantly found in T. cruzi parasites, has been shown to interfere with cardiomyocyte apoptosis through activation of the NFא B and PI3K/Akt and MEK1/ERK1/2 pathways in the host cell, which lead to increased expression of antiapoptotic Bcl2 molecules and increased arginase expression [67]. It is therefore conceivable that the crosstalk of the intracellular parasite with the signaling pathways of these structural cells might negatively impact the production of chemotactic molecules.

A deficit in intracellular parasite sensing, although important, is insufficient to guarantee T. cruzi evasion in the chronically infected heart. This is the case given that, sooner or later, an undetected amastigote nest will spontaneously disrupt, releasing extracellular parasites that, after detection by antibodies, will cause complement and resident macrophages to generate mediators for leukocyte recruitment. Thus, for long-term parasite perpetuation to occur, it is predictable that a small fraction of nest-released trypomastigotes will reinvade relatively distant cardiomyocytes, where they may remain unnoticed and out of the reach of cytotoxic $\mathrm{CD} 8^{+} \mathrm{T}$ cells in the newly formed infiltrate.

As stated above, defective parasite sensing by cardiomyocytes could be an adaptive process of the heart tissue that develops with the length of infection. This process most likely exhibits a great degree of variability, reflecting the genetics of the host and the parasite. Moreover, its occurrence in the cardiac tissue is not surprising because the heart is a vital organ that must have special mechanisms designed to protect its integrity.

\section{Local Parasite Destruction versus Immunopathology}

Theoretically, if two hosts are unable to control tissue parasites, the one with a greater local inflammatory response will pay a higher price by provoking greater damage of the infected tissue [68]. Therefore, if defective local sensing occurs in CCC patients, strong local immune responses would clearly represent a detrimental factor in the induction of pathology, thus explaining the reported associations between high levels of cardiac dysfunction and genotypes associated with high reactivity $[69,70]$. Furthermore, the inverse correlation observed in CCC patients between the intensity of electrocardiogram abnormalities and IL-10 plasma levels [59] reinforces this view. That is, to respond strongly when there is a gap in local T. cruzi control clearly represents a deleterious manner of dealing with the parasite.

Entering into a persistent cycle of an intense local effector response with no resolution is, to a certain extent, a form of autoaggression, considering the high price paid by the organism in terms of tissue damage. It is not, however, an aggression specifically directed against self-antigens but the unwanted price for unceasingly attempting to completely eliminate a small number of parasites that persist in a fraction of chronically infected individuals. Paradoxically, to protect heart integrity, cardiomyocytes may allow parasite persistence, which indirectly results in tissue damage every time a nest breaks open and new leukocyte infiltrates are formed.

A deficit in the interaction of T. cruzi parasites with tissue structural cells could also be involved in parasite persistence at locations other than the heart. This could occur in any infected patient, independent of whether the infection is cardiac, digestive, or indeterminate. In contrast to the heart, however, in many of these locations, the bystander tissue damage resulting from the persistent immune reaction against parasites might not be sufficient to compromise the function of the infected tissue.

\section{Future Perspectives}

While extensive research has deciphered the local and systemic immune responses of chronically $T$. cruzi-infected hosts in the last two decades, future studies will need to focus on the in vivo interaction of parasites with structural cells, in both the heart and other tissues. Although these studies currently face great technical challenges, they will be of great importance to improve our knowledge about Chagas disease pathology.

\section{Disclosure}

Figure 3 is reprinted with permission from Elsevier from Microbes and Infection [64].

\section{Conflict of Interests}

The authors declare that there is no conflict of interests regarding the publication of this paper.

\section{Acknowledgments}

The authors extend their thanks to Rogério Silva do Nascimento and Bernardo Paulo Albe for providing technical support. Financial support was provided by Grants FAPESP 2013/08199-0 and CNPq 303269/2010-3.

\section{References}

[1] OMS, "Chagas disease (American trypanosomiasis)," Fact Sheet 340, 2013, http://www.who.int/mediacentre/factsheets/fs340/ en/index.html

[2] J. R. Coura and P. A. Vĩas, "Chagas disease: a new worldwide challenge," Nature, vol. 465, no. 7301, pp. S6-S7, 2010.

[3] A. L. Bombeiro, L. A. Gonçalves, C. Penha-Gonçalves et al., "IL-12p40 deficiency leads to uncontrolled Trypanosoma cruzi dissemination in the spinal cord resulting in neuronal death and motor dysfunction," PLoS ONE, vol. 7, no. 11, Article ID e49022, 2012. 
[4] S. G. Andrade and Z. A. Andrade, "Pathology of prolonged experimental Chagas' disease," Revista do Instituto de Medicina Tropical de Sao Paulo, vol. 10, no. 3, pp. 180-187, 1968.

[5] R. P. Laguens, P. C. Meckert, and R. J. Gelpi, "Chronic Chagas disease in the mouse. I. Electrocardiographic and morphological patterns of the cardiopathy.," Medicina B, vol. 41, no. 1, pp. 35-39, 1981.

[6] F. G. Araujo, "Development of resistance to Trypanosoma cruzi in mice depends on a viable population of L3T4+ (CD4+) T lymphocytes," Infection and Immunity, vol. 57, no. 7, pp. 22462248, 1989.

[7] R. L. Tarleton, B. H. Koller, A. Latour, and M. Postan, "Susceptibility of $\beta_{2}$-microglobulin-deficient mice to Trypanosoma cruzi infection," Nature, vol. 356, no. 6367, pp. 338-340, 1992.

[8] A. M. Rodriguez, F. Santoro, D. Afchain, H. Bazin, and A. Capron, "Trypanosoma cruzi infection in B-cell-deficient rats," Infection and Immunity, vol. 31, no. 2, pp. 524-529, 1981.

[9] L. F. Umekita, H. A. Takehara, and I. Mota, "Role of the antibody Fc in the immune clearance of Trypanosoma cruzi," Immunology Letters, vol. 17, no. 1, pp. 85-89, 1988.

[10] I. Mota and L. F. Umekita, "The effect of C3 depletion on the clearance of Trypanosoma cruzi induced by IgG antibodies," Immunology Letters, vol. 21, no. 3, pp. 223-226, 1989.

[11] L. R. Sardinha, T. Mosca, R. M. Elias et al., "The liver plays a major role in clearance and destruction of blood trypomastigotes in Trypanosoma cruzichronically infected mice," PLoS Neglected Tropical Diseases, vol. 4, no. 1, article e578, 2010.

[12] F. Cardillo, J. C. Voltarelli, S. G. Reed, and J. S. Silva, "Regulation of Trypanosoma cruzi infection in mice by gamma interferon and interleukin 10: role of NK cells," Infection and Immunity, vol. 64, no. 1, pp. 128-134, 1996.

[13] L. R. Sardinha, R. M. Elias, T. Mosca et al., "Contribution of $\mathrm{NK}, \mathrm{NK} \mathrm{T}, \gamma \delta \mathrm{T}$, and $\alpha \beta \mathrm{T}$ cells to the gamma interferon response required for liver protection against Trypanosoma cruzi," Infection and Immunity, vol. 74, no. 4, pp. 2031-2042, 2006.

[14] R. E. McCabe, S. G. Meagher, and B. T. Mullins, "Endogenous interferon- $\gamma$, macrophage activation, and murine host defense against acute infection with Trypanosoma cruzi," Journal of Infectious Diseases, vol. 163, no. 4, pp. 912-915, 1991.

[15] R. T. Gazzinelli, I. P. Oswald, S. Hieny, S. L. James, and A. Sher, "The microbicidal activity of interferon- $\gamma$-treated macrophages against Trypanosoma cruzi involves an Larginine-dependent, nitrogen oxide-mediated mechanism inhibitable by interleukin-10 and transforming growth factor$\beta$," European Journal of Immunology, vol. 22, no. 10, pp. 2501-2506, 1992.

[16] H. P. Low, M. A. M. Santos, B. Wizel, and R. L. Tarleton, "Amastigote surface proteins of Trypanosoma cruzi are targets for CD8 ${ }^{+}$CTL," Journal of Immunology, vol. 160, no. 4, pp. 18171823, 1998.

[17] K. A. Joiner, W. D. daSilva, M. T. Rimoldi, C. H. Hammer, A. Sher, and T. L. Kipnis, "Biochemical characterization of a factor produced by trypomastigotes of Trypanosoma cruzi that accelerates the decay of complement C3 convertases," The Journal of Biological Chemistry, vol. 263, no. 23, pp. 11327-11335, 1988.

[18] N. Nogueira and Z. Cohn, "Trypanosoma cruzi: mechanism of entry and intracellular fate in mammalian cells," Journal of Experimental Medicine, vol. 143, no. 6, pp. 1402-1420, 1976.
[19] H. Castro and A. M. Tomás, "Peroxidases of trypanosomatids," Antioxidants and Redox Signaling, vol. 10, no. 9, pp. 1593-1606, 2008.

[20] L. Piacenza, M. P. Zago, G. Peluffo, M. N. Alvarez, M. A. Basombrio, and R. Radi, "Enzymes of the antioxidant network as novel determiners of Trypanosoma cruzi virulence," International Journal for Parasitology, vol. 39, no. 13, pp. 1455-1464, 2009.

[21] L. Piacenza, M. N. Alvarez, G. Peluffo, and R. Radi, "Fighting the oxidative assault: the Trypanosoma cruzi journey to infection," Current Opinion in Microbiology, vol. 12, no. 4, pp. 415-421, 2009.

[22] M. A. S. Campos, I. C. Almeida, O. Takeuchi et al., "Activation of toll-like receptor-2 by glycosylphosphatidylinositol anchors from a protozoan parasite," Journal of Immunology, vol. 167, no. 1, pp. 416-423, 2001.

[23] A. Oliveira, J. R. Peixoto, L. B. de Arrada et al., "Expression of functional TLR4 confers proinflammatory responsiveness to Trypanosoma cruzi glycoinositolphospholipids and higher resistance to infection with T. cruzi," Journal of Immunology, vol. 173, no. 9, pp. 5688-5696, 2004.

[24] B. C. Caetano, B. B. Carmo, M. B. Melo et al., "Requirement of UNC93B1 reveals a critical role for TLR7 in host resistance to primary infection with Trypanosoma cruzi," Journal of Immunology, vol. 187, no. 4, pp. 1903-1911, 2011.

[25] G. K. Silva, F. R. S. Gutierrez, P. M. M. Guedes et al., "Cutting edge: nucleotide-binding oligomerization domain 1-dependent responses account for murine resistance against Trypanosoma cruzi infection," Journal of Immunology, vol. 184, no. 3, pp. 1148$1152,2010$.

[26] G. K. Silva, R. S. Costa, T. N. Silveira et al., "Apoptosis-associated speck-like protein containing a caspase recruitment domain inflammasomes mediate IL-1 $\beta$ response and host resistance to Trypanosoma cruzi infection," The Journal of Immunology, vol. 191, no. 6, pp. 3373-3383, 2013.

[27] V. M. Gon $\tau$ alves, K. C. Matteucci, C. L. Buzzo et al., "NLRP3 controls Trypanosoma cruzi infection through a caspase-1dependent IL-1R-independent NO production," PLoS Neglected Tropical Diseases, vol. 7, no. 10, article e2469, 2013.

[28] S. P. Kurup and R. L. Tarleton, "Perpetual expression of PAMPs necessary for optimal immune control and clearance of a persistent pathogen," Nature Communications, vol. 4, article 2616, 2013.

[29] F. Tzelepis, B. C. G. De Alencar, M. L. O. Penido, R. T. Gazzinelli, P. M. Persechini, and M. M. Rodrigues, "Distinct kinetics of effector $\mathrm{CD}^{+}$cytotoxic T cells after infection with Trypanosoma cruzi in naive or vaccinated mice," Infection and Immunity, vol. 74, no. 4, pp. 2477-2481, 2006.

[30] J. R. Vasconcelos, O. Bruña-Romero, A. F. Araújo et al., "Pathogen-induced proapoptotic phenotype and high CD95 (Fas) expression accompany a suboptimal CD8+ T-cell response: reversal by adenoviral vaccine," PLoS Pathogens, vol. 8, no. 5, Article ID e1002699, 2012.

[31] F. Tzelepis, B. C. G. de Alencar, M. L. O. Penido et al., "Infection with Trypanosoma cruzi restricts the repertoire of parasitespecific CD8+ T cells leading to immunodominance," Journal of Immunology, vol. 180, no. 3, pp. 1737-1748, 2008.

[32] M. C. Albareda, G. C. Olivera, S. A. Laucella et al., "Chronic human infection with Trypanosoma cruzi drives $\mathrm{CD}^{+}{ }^{+} \mathrm{T}$ cells to immune senescence," The Journal of Immunology, vol. 183, no. 6, pp. 4103-4108, 2009. 
[33] J. K. Leavey and R. L. Tarleton, "Cutting edge: dysfunctional $\mathrm{CD} 8+\mathrm{T}$ cells reside in nonlymphoid tissues during chronic Trypanosoma cruzi infection," Journal of Immunology, vol. 170, no. 5, pp. 2264-2268, 2003.

[34] D. L. Martin, D. B. Weatherly, S. A. Laucella et al., "CD8+ T-cell responses to Trypanosoma cruzi are highly focused on strainvariant trans-sialidase epitopes," PLoS Pathogens, vol. 2, no. 8, pp. 731-740, 2006.

[35] D. L. Martin, M. Postan, P. Lucas, R. Gress, and R. L. Tarleton, "TGF- $\beta$ regulates pathology but not tissue $\mathrm{CD}^{+} \mathrm{T}$ cell dysfunction during experimental Trypanosoma cruzi infection," European Journal of Immunology, vol. 37, no. 10, pp. 2764-2771, 2007.

[36] M. C. Albareda, A. M. de Rissio, G. Tomas et al., "Polyfunctional $\mathrm{T}$ cell responses in children in early stages of chronic Trypanosoma cruzi infection contrast with monofunctional responses of long-term infected adults," PLoS Neglected Tropical Diseases, vol. 7, no. 12, article e2575, 2013.

[37] F. R. Gutierrez, F. S. Mariano, C. J. Oliveira et al., "Regulation of Trypanosoma cruzi-induced myocarditis by programmed death cell receptor 1," Infection and Immunity, vol. 79, no. 5, pp. 18731881, 2011.

[38] C. M. Ayo, M. M. Dalalio, J. E. Visentainer et al., "Genetic susceptibility to Chagas disease: an overview about the infection and about the association between disease and the immune response genes," BioMed Research International, vol. 2013, Article ID 284729, 13 pages, 2013.

[39] C. Sanoja, S. Carbajosa, M. Fresno, and N. Gironès, "Analysis of the dynamics of infiltrating $\mathrm{CD} 4^{+} \mathrm{T}$ cell subsets in the heart during experimental Trypanosoma cruzi infection," PLoS ONE, vol. 8, no. 6, Article ID e65820, 2013.

[40] J. C. Pinto Dias, "The indeterminate form of human chronic Chagas' disease. A clinical epidemiological review," Revista da Sociedade Brasileira de Medicina Tropical, vol. 22, no. 3, pp. 147156, 1989.

[41] R. Ribeiro-Dos-Santos, J. O. Mengel, E. Postol et al., "A heartspecific CD4+ T-cell line obtained from a chronic chagasic mouse induces carditis in heart-immunized mice and rejection of normal heart transplants in the absence of Trypanosoma cruzi," Parasite Immunology, vol. 23, no. 2, pp. 93-101, 2001.

[42] E. Cunha-Neto, M. Duranti, A. Gruber et al., "Autoimmunity in Chagas disease cardiopathy: biological relevance of a cardiac myosin-specific epitope crossreactive to an immunodominant Trypanosoma cruzi antigen," Proceedings of the National Academy of Sciences of the United States of America, vol. 92, no. 8, pp. 3541-3545, 1995.

[43] L. Sterin-Borda and E. Borda, "Role of neurotransmitter autoantibodies in the pathogenesis of chagasic peripheral dysautonomia," Annals of the New York Academy of Sciences, vol. 917, pp. 273-280, 2000.

[44] M. D. L. Higuchi, M. M. Reis, V. D. Aiello et al., "Association of an increase in CD8+ T cells with the presence of Trypanosoma cruzi antigens in chronic, human, chagasic myocarditis," American Journal of Tropical Medicine and Hygiene, vol. 56, no. 5, pp. 485-489, 1997.

[45] E. M. Jones, D. G. Colley, S. Tostes, E. R. Lopes, C. L. VnencakJones, and T. L. McCurley, "Amplification of a Trypanosoma cruzi DNA sequence from inflammatory lesions in human chagasic cardiomyopathy," American Journal of Tropical Medicine and Hygiene, vol. 48, no. 3, pp. 348-357, 1993.

[46] A. R. Vago, A. M. Macedo, S. J. Adad, D. D’Avila Reis, and R. Correa-Oliveira, "PCR detection of Trypanosoma cruzi DNA in oesophageal tissues of patients with chronic digestive Chagas' disease," The Lancet, vol. 348, no. 9031, pp. 891-892, 1996.

[47] C. R. F. Marinho, D. Z. Bucci, M. L. Z. Dagli et al., "Pathology affects different organs in two mouse strains chronically infected by a Trypanosoma cruzi clone: a model for genetic studies of Chagas'disease," Infection and Immunity, vol. 72, no. 4, pp. 2350-2357, 2004.

[48] R. L. Tarleton, "Parasite persistence in the aetiology of Chagas disease," International Journal for Parasitology, vol. 31, no. 5-6, pp. 550-554, 2001.

[49] M. D. Lewis, A. F. Francisco, M. C. Taylor et al., "Bioluminescence imaging of chronic Trypanosoma cruzi infections reveals tissue-specific parasite dynamics and heart disease in the absence of locally persistent infection," Cellular Microbiology, 2014.

[50] V. de Paula Antunes Teixeira, V. Hial, R. A. da Silva Gomes et al., "Correlation between adrenal central vein parasitism and heart fibrosis in chronic chagasic myocarditis," American Journal of Tropical Medicine and Hygiene, vol. 56, no. 2, pp. 177-180, 1997.

[51] T. P. Combs, S. Mukherjee, C. J. G. De Almeida et al., “The adipocyte as an important target cell for Trypanosoma cruzi infection," The Journal of Biological Chemistry, vol. 280, no. 25, pp. 24085-24094, 2005.

[52] A. V. Ferreira, M. Segatto, Z. Menezes et al., "Evidence for Trypanosoma cruzi in adipose tissue in human chronic Chagas disease," Microbes and Infection, vol. 13, no. 12-13, pp. 1002-1005, 2011.

[53] B. Zingales, S. G. Andrade, M. R. S. Briones et al., "A new consensus for Trypanosoma cruzi intraspecific nomenclature: second revision meeting recommends TcI to TcVI," Memorias do Instituto Oswaldo Cruz, vol. 104, no. 7, pp. 1051-1054, 2009.

[54] L. G. Nogueira, R. H. B. Santos, B. M. Ianni et al., "Myocardial chemokine expression and intensity of myocarditis in Chagas cardiomyopathy are controlled by polymorphisms in CXCL9 and CXCL10," PLoS Neglected Tropical Diseases, vol. 6, no. 10, Article ID e1867, 2012.

[55] D. B. Rocha Rodrigues, M. A. dos Reis, A. Romano et al., "In situ expression of regulatory cytokines by heart inflammatory cells in Chagas' disease patients with heart failure," Clinical and Developmental Immunology, vol. 2012, Article ID 361730, 7 pages, 2012.

[56] E. Cunha-Neto, V. J. Dzau, P. D. Allen et al., "Cardiac gene expression profiling provides evidence for cytokinopathy as a molecular mechanism in Chagas'disease cardiomyopathy," The American Journal of Pathology, vol. 167, no. 2, pp. 305-313, 2005.

[57] M. M. Reis, M. D. L. Higuchi, L. A. Benvenuti et al., "An in situ quantitative immunohistochemical study of cytokines and IL-2R+ in chronic human chagasic myocarditis: correlation with the presence of myocardial Trypanosoma cruzi antigens," Clinical Immunology and Immunopathology, vol. 83, no. 2, pp. 165-172, 1997.

[58] J. C. Silverio, I. R. Pereira, M. D. C. Cipitelli et al., "CD8+ Tcells expressing interferon gamma or perforin play antagonistic roles in heart injury in experimental Trypanosoma cruzi-elicited cardiomyopathy," PLoS Pathogens, vol. 8, no. 4, Article ID e1002645, 2012.

[59] G. R. Sousa, J. A. Gomes, R. C. Fares et al., "Plasma cytokine expression is associated with cardiac morbidity in chagas disease," PLoS ONE, vol. 9, no. 3, Article ID e87082, 2014.

[60] J. A. S. Gomes, L. M. G. Bahia-Oliveira, M. O. C. Rocha, O. A. Martins-Filho, G. Gazzinelli, and R. Correa-Oliveira, "Evidence 
that development of severe cardiomyopathy in human Chagas' disease is due to a Th1-specific immune response," Infection and Immunity, vol. 71, no. 3, pp. 1185-1193, 2003.

[61] C. Poveda, M. Fresno, N. Gironés et al., "Cytokine profiling in Chagas disease: towards understanding the association with infecting Trypanosoma cruzi discrete typing units (A BENEFIT TRIAL SubStudy)," PLoS ONE, vol. 9, no. 3, Article ID e0091154, 2014.

[62] F. F. de Araújo, R. Corrêa-Oliveira, M. O. C. Rocha et al., "Foxp $3^{+} \mathrm{CD}^{25}$ high $\mathrm{CD} 4^{+}$regulatory T cells from indeterminate patients with Chagas disease can suppress the effector cells and cytokines and reveal altered correlations with disease severity," Immunobiology, vol. 217, no. 8, pp. 768-777, 2012.

[63] W. O. Dutra, C. A. S. Menezes, F. N. A. Villani et al., "Cellular and genetic mechanisms involved in the generation of protective and pathogenic immune responses in human Chagas disease," Memorias do Instituto Oswaldo Cruz, vol. 104, no. 1, pp. 208-218, 2009.

[64] C. R. F. Marinho, L. N. Nuñez-Apaza, K. R. Bortoluci et al., "Infection by the Sylvio X10/4 clone of Trypanosoma cruzi: relevance of a low-virulence model of Chagas' disease," Microbes and Infection, vol. 11, no. 13, pp. 1037-1045, 2009.

[65] G. Vianna, "Contribuição para o estudo da Anatomia Patológica da Molestia de Carlos Chagas," Memorias do Instituto Oswaldo Cruz, vol. 3, p. 276, 1911.

[66] F. S. Machado, G. A. Martins, J. C. S. Aliberti, F. L. A. C. Mestriner, F. Q. Cunha, and J. S. Silva, "Trypanosoma cruziinfected cardiomyocytes produce chemokines and cytokines that trigger potent nitric oxide-dependent trypanocidal activity," Circulation, vol. 102, no. 24, pp. 3003-3008, 2000.

[67] M. D. P. Aoki, R. C. Cano, A. V. Pellegrini et al., "Different signaling pathways are involved in cardiomyocyte survival induced by a Trypanosoma cruzi glycoprotein," Microbes and Infection, vol. 8, no. 7, pp. 1723-1731, 2006.

[68] L. Råberg, D. Sim, and A. F. Read, "Disentangling genetic variation for resistance and tolerance to infectious diseases in animals," Science, vol. 318, no. 5851, pp. 812-814, 2007.

[69] R. Ramasawmy, K. C. Fae, E. Cunha-Neto et al., "Polymorphisms in the gene for lymphotoxin- $\alpha$ predispose to chronic chagas cardiomyopathy," Journal of Infectious Diseases, vol. 196, no. 12, pp. 1836-1843, 2007.

[70] G. Zafra, C. Morillo, J. Martín, A. González, and C. I. González, "Polymorphism in the $3^{\prime}$ UTR of the IL12B gene is associated with Chagas' disease cardiomyopathy," Microbes and Infection, vol. 9, no. 9, pp. 1049-1052, 2007. 


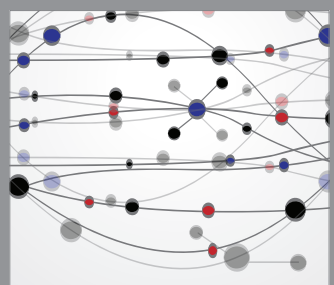

The Scientific World Journal
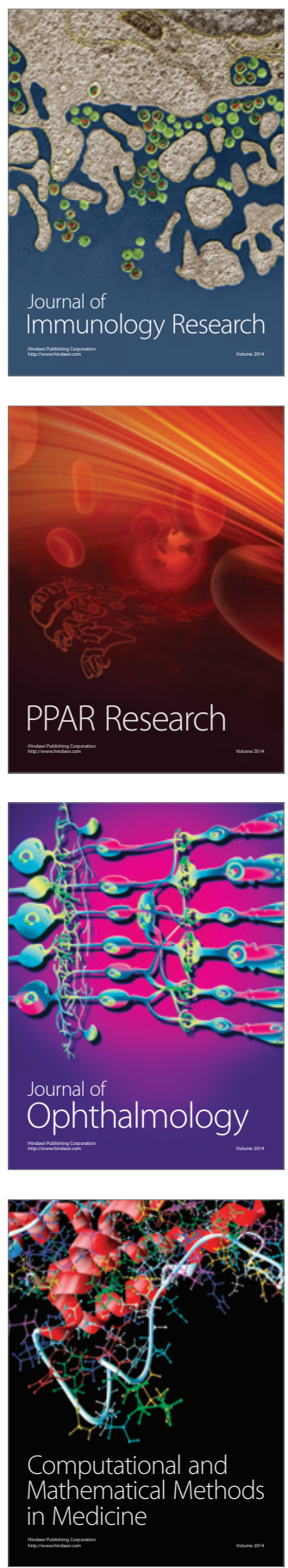

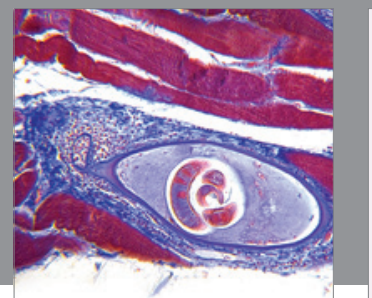

Gastroenterology

Research and Practice
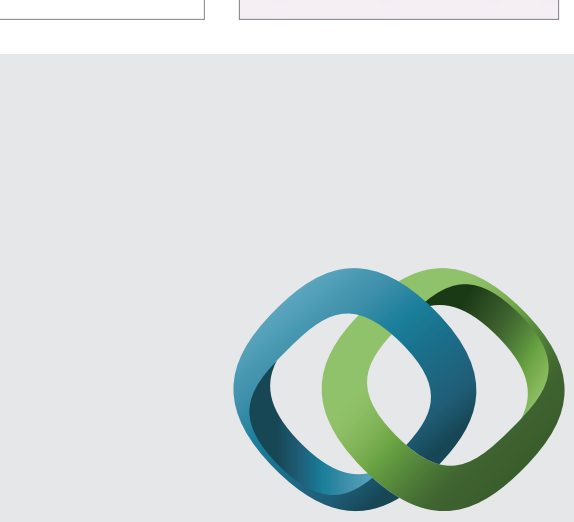

\section{Hindawi}

Submit your manuscripts at

http://www.hindawi.com
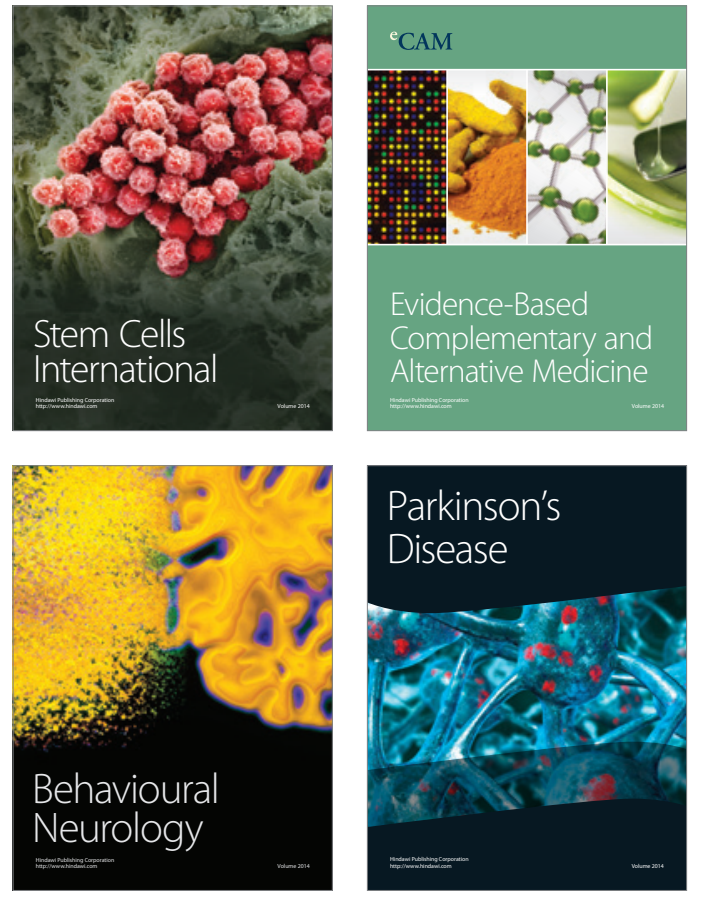
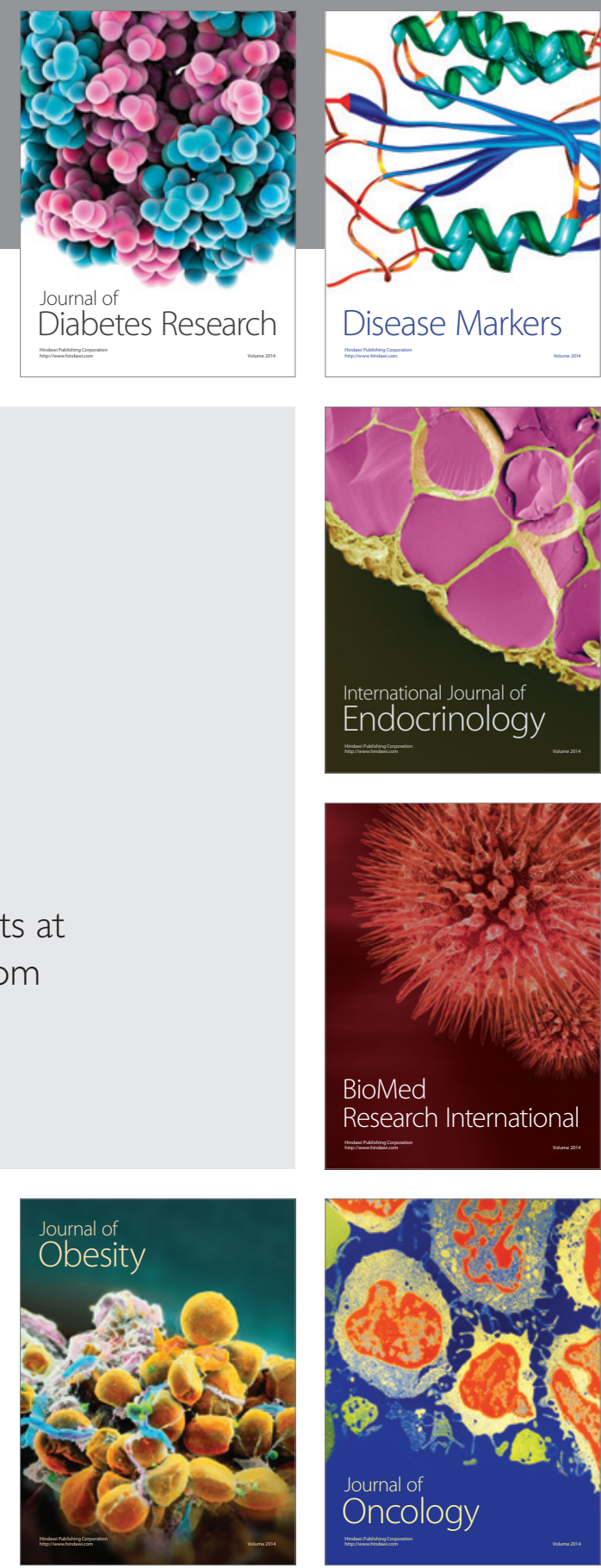

Disease Markers
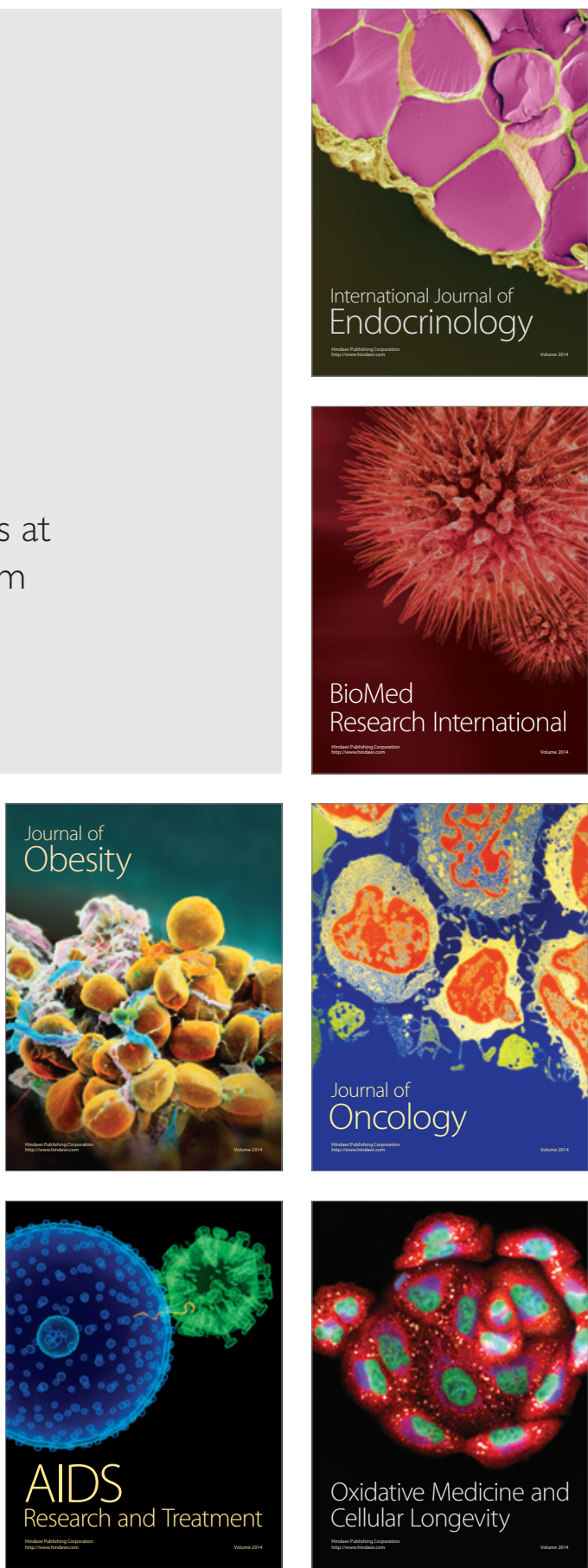